Supporting Information

\title{
Revealing Facet Effects of Palladium Nanocrystals on Electrochemical Biosensing
}

Yang Yang, ${ }^{\dagger}$ Jiachang Zeng, ${ }^{+}$Yijin Shu ${ }^{\dagger}$ and Qingsheng $\mathrm{Gao}^{*,+}$

Department of Chemistry, College of Chemistry and Materials Science, Jinan University, Guangzhou 510632, P. R. China

E-mail: tqsgao@jnu.edu.cn (Qingsheng Gao) 


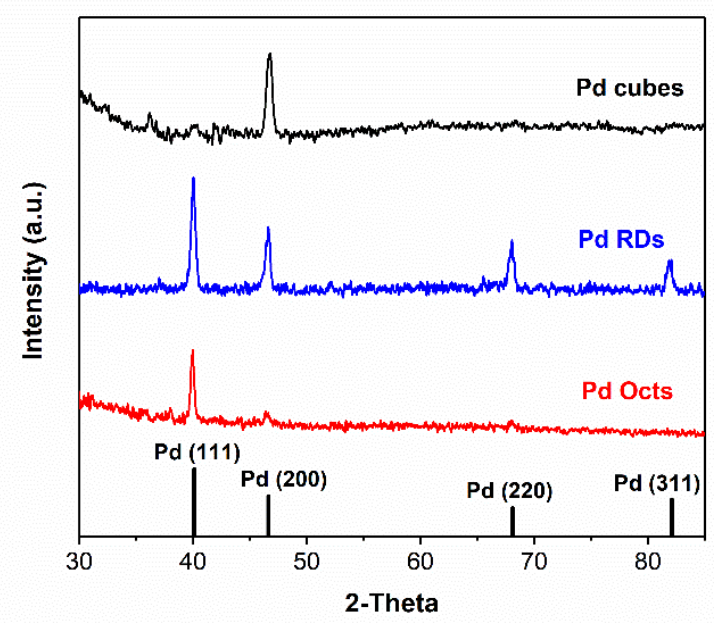

Figure S1. XRD patterns of Pd cubes (black), RDs (blue) and Octs (red). Pd Cubes and Pd Octs mainly exhibit the strongest diffraction peaks ascribed to the (200) and (111) planes, respectively. By contrast, Pd RDs show the four peaks of Pd, because it was very difficult to obtain an ordered orientation for Pd RDs at flat and polished glass substrate by self-assembly, in comparison to cube and Pd Octs.
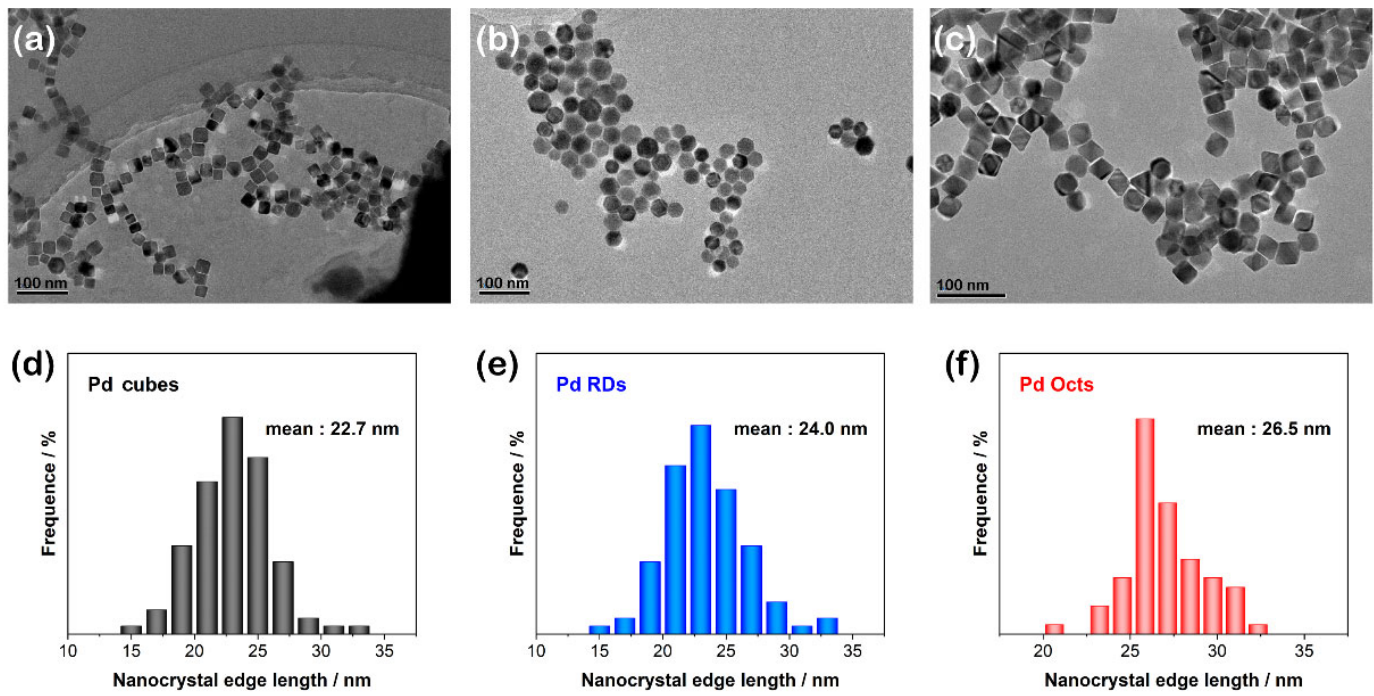

Figure S2. TEM images of (a) Pd cubes, (b) RDs and (c) Octs at the scale bar of $100 \mathrm{~nm}$ and nanocrystal edge length distribution histograms for Pd (d) cubes, (e) RDs and (f) Octs. 

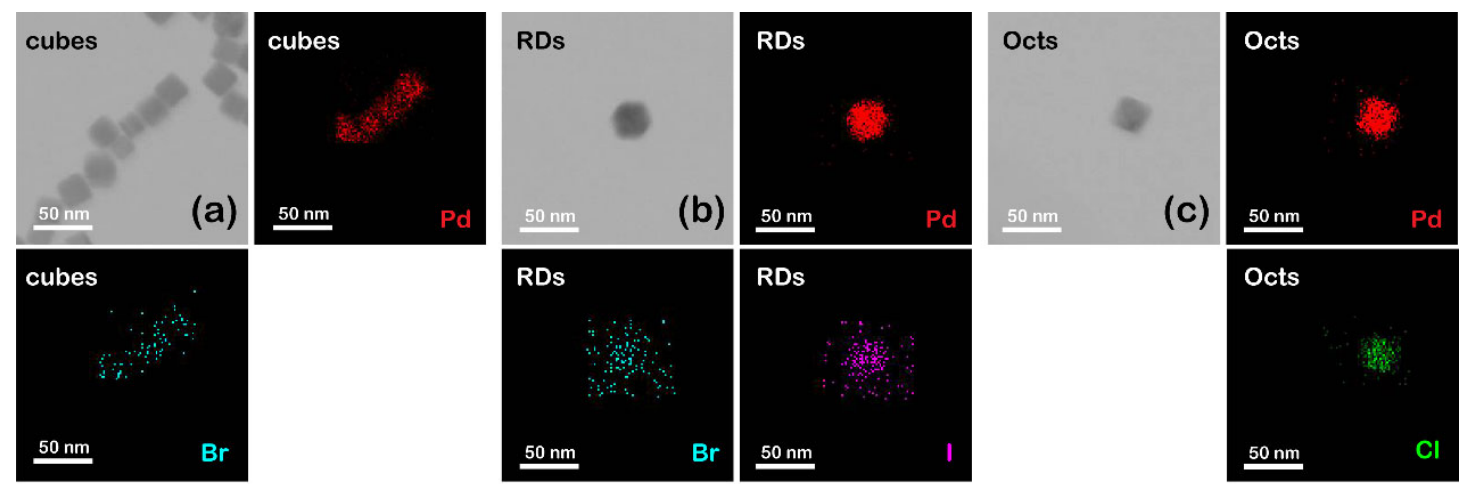

Figure S3. Bright-field STEM images of Pd cubes (a), RDs (b) and Octs (c) at the scale bar of $50 \mathrm{~nm}$ along with corresponding elemental mapping.
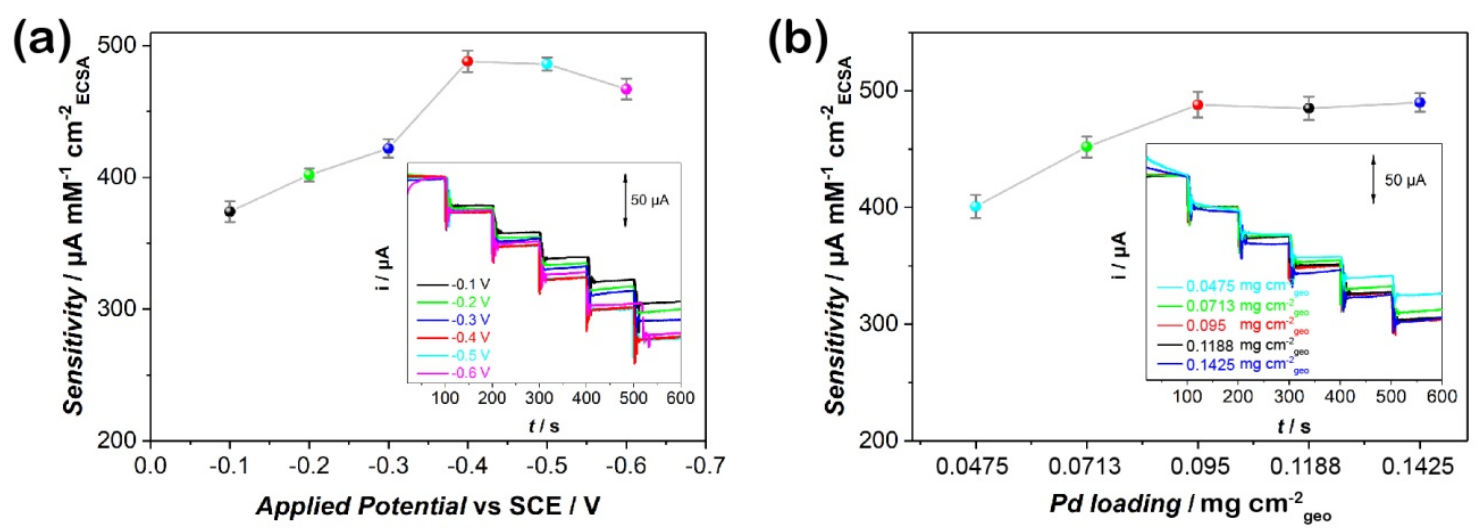

Figure S4. Effects of (a) applied potential and (b) Pd loading on the sensitivity of Pd cubes modified RDE in $0.1 \mathrm{M}$ PB solution. The insets of $\mathrm{a}$ and $\mathrm{b}$ are the corresponding amperometric i- $t$ curves in response to the successive addition of $100 \mu \mathrm{M} \mathrm{H}_{2} \mathrm{O}_{2}$. 


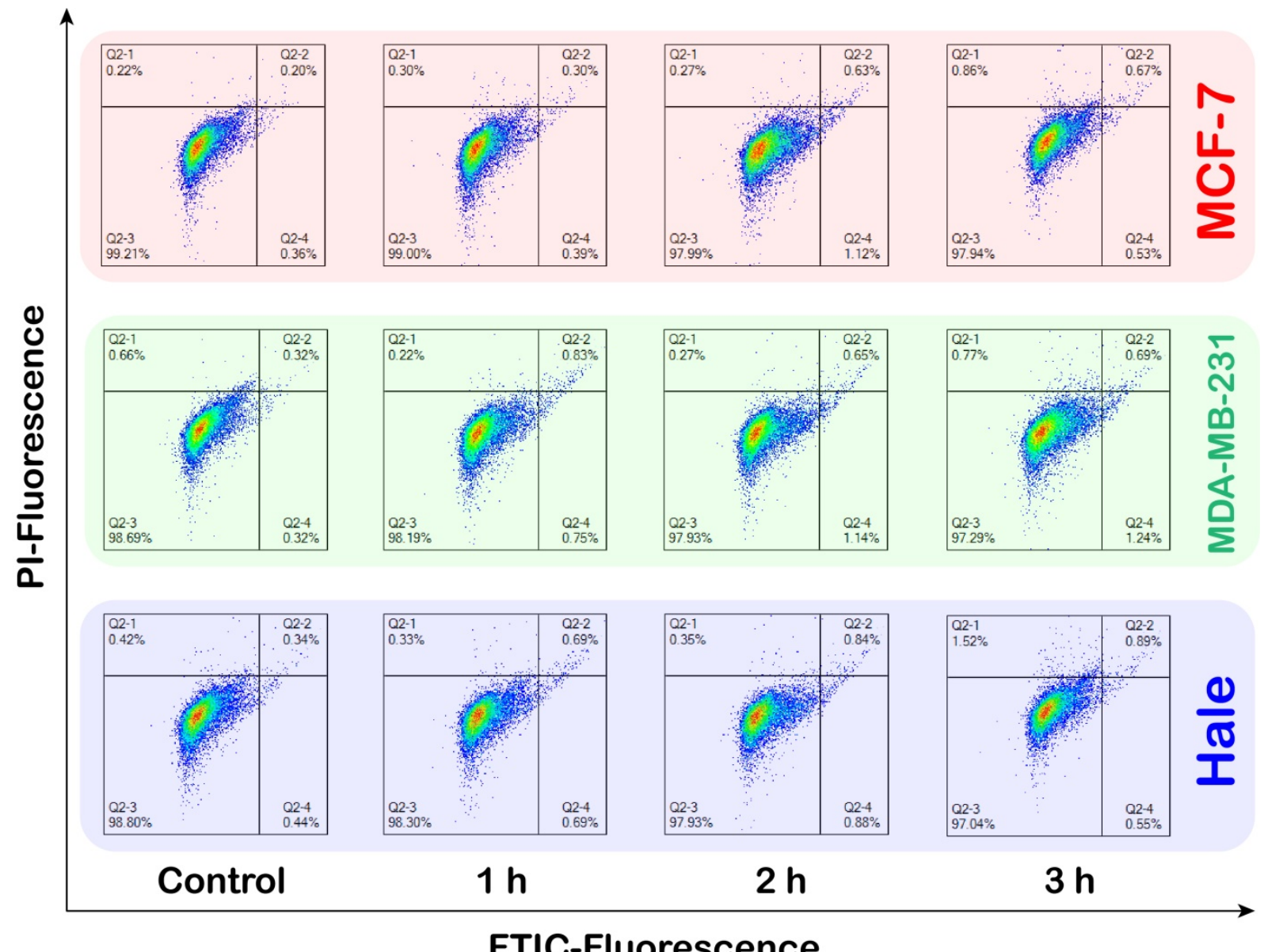

FTIC-Fluorescence

Figure S5. Measurement of MCF-7, MDA-MB-231 and Hale cells apoptosis by AV/PI double staining. The Pd cubes modified RDE fail to induce significant apoptosis in these three group, indicating that the Pd cubes have minimal cytotoxicity. 
(a)
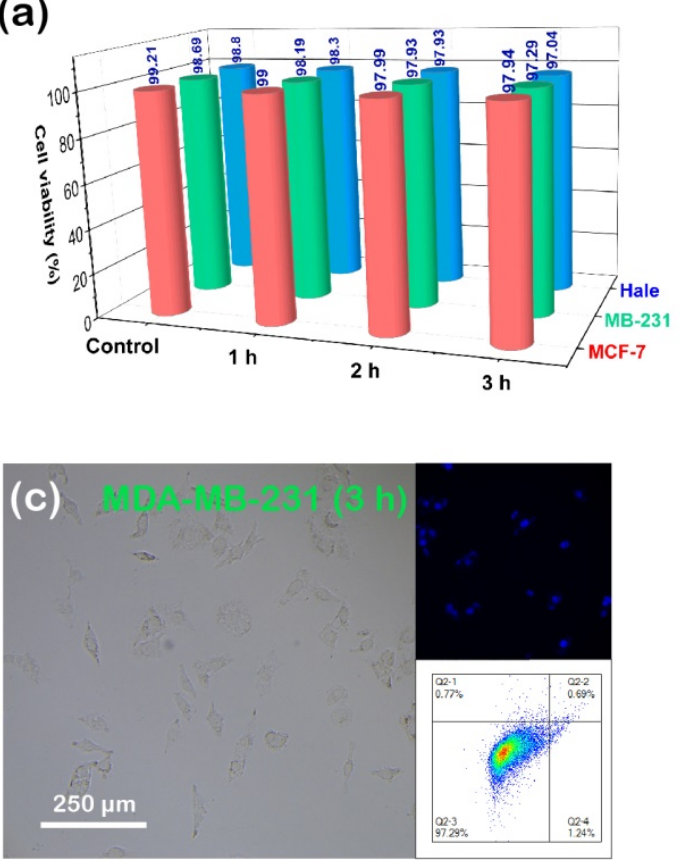
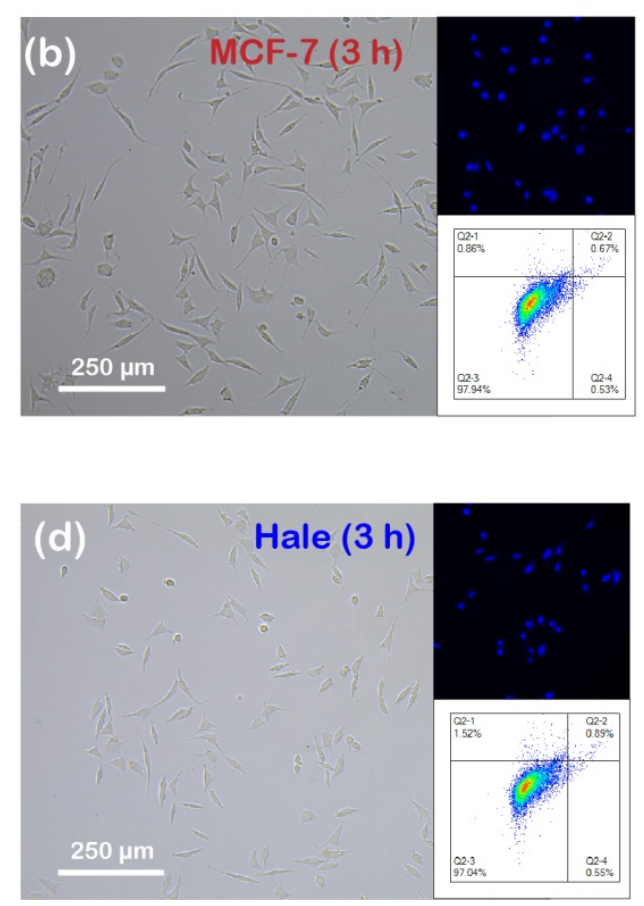

Figure S6. (a) Quantitative cell viability results of AV/PI double staining for MCF-7, MDA-MB-231 and Hale cells. All of the cell viabilities are $>90 \%$ when the cancer cells being incubated with Pd cubes for 3 hours. In addition, bright-field, dark-field fluorescent images and cells apoptosis measurement by AV/PI double staining of MCF-7 (b), MDA-MB-231 (c) and Hale (d) cells, which demonstrate all of these three cells remain healthy when incubated with Pd cubes for 3 hours.
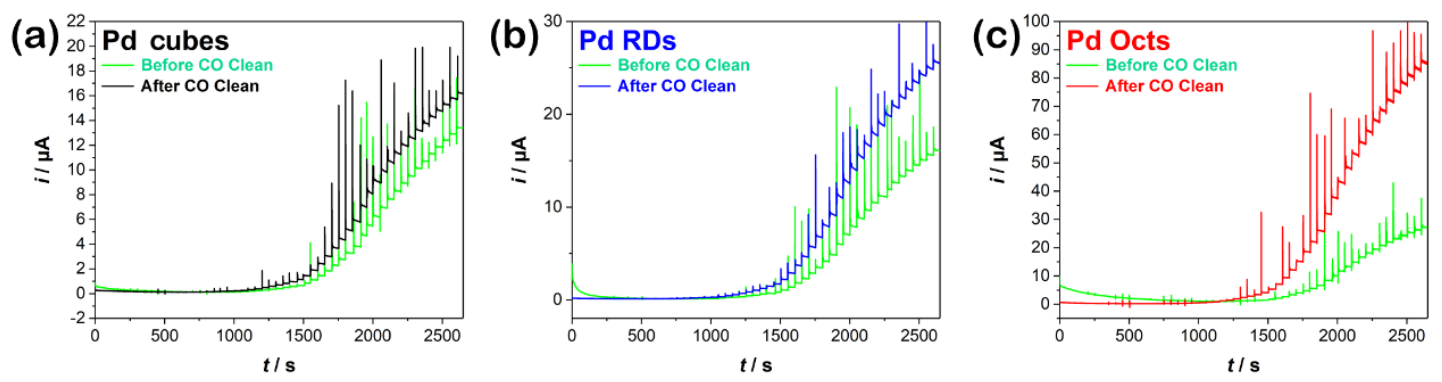

Figure S7. Amperometric response of Pd (a) cubes, (b) Pd RDs and (c) Pd Octs modified RDEs to the dropwise addition of glucose in $0.1 \mathrm{M} \mathrm{KOH}$ solution at $1600 \mathrm{rpm}$ before and after CO surface cleaning treatment (working potential: $0.1 \mathrm{~V}$ vs. SCE). 


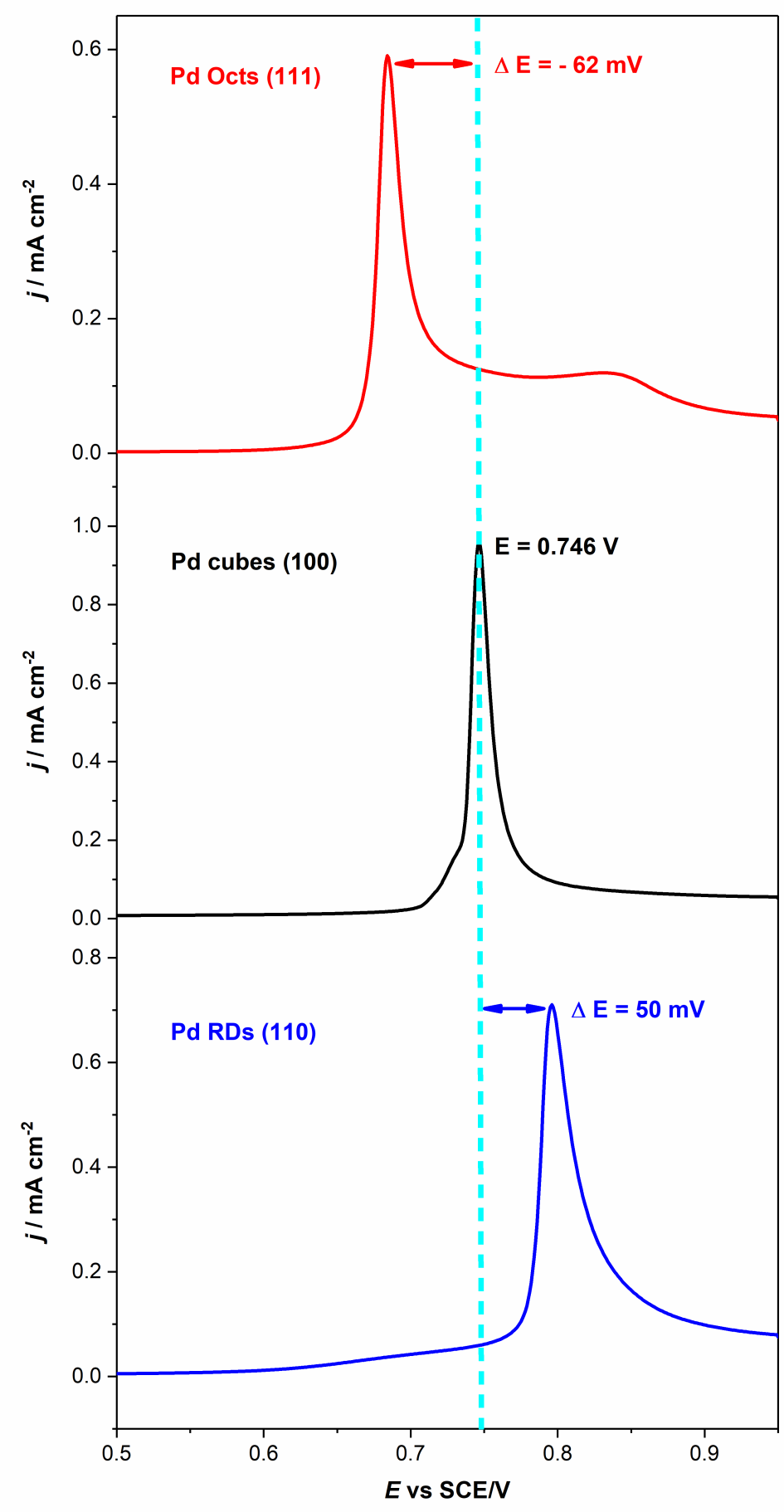

Figure S8. CO stripping curves obtained from the three types of $\mathrm{Pd} \mathrm{NCs}$ in $0.5 \mathrm{M} \mathrm{H}_{2} \mathrm{SO}_{4}$. Scan rate: $50 \mathrm{mV} \mathrm{s}^{-1}$. 
Table S1. Comparison of electrochemical $\mathrm{H}_{2} \mathrm{O}_{2}$ sensors reported previously with the Pd cubes

\begin{tabular}{|c|c|c|c|c|}
\hline & $\begin{array}{c}\text { Sensitivity } \\
\left(\mu \mathrm{A} \mathrm{mM}^{-1} \mathrm{~cm}_{\text {geo }^{-2}}\right)\end{array}$ & $\begin{array}{l}\text { Linear range } \\
\qquad(\mu \mathrm{M})\end{array}$ & $\begin{array}{l}\mathrm{LOD} \\
(\mu \mathrm{M})\end{array}$ & References \\
\hline Pd cubes & 1434 & $0.6 \sim 51999$ & 0.6 & This work \\
\hline Pd-SnO $2 @ M W C N T s$ & 46.1 & $10 \sim 50000$ & 2.8 & 1 \\
\hline \multirow{2}{*}{$\mathrm{Nf} / \mathrm{Pd} @ \mathrm{CeO}_{2} \mathrm{NH}_{2}$} & 440.72 & $1 \sim 3276$ & \multirow{2}{*}{0.47} & \multirow{2}{*}{2} \\
\hline & 852.65 & $3276 \sim 17500$ & & \\
\hline Pd/PDDA/PGR & 816.7 & $2 \sim 1672$ & 0.9 & 3 \\
\hline $\mathrm{Fe} @ \mathrm{Pt} / \mathrm{C}$ & 218.97 & $2.5 \sim 41605$ & 0.75 & 4 \\
\hline GR-AuNRs & 389.2 & 305000 & 10 & 5 \\
\hline $\mathrm{PtNi} / \mathrm{CeO}_{2} / \mathrm{NCNFs}$ & 345.0 & $0.5 \sim 15000$ & 0.025 & 6 \\
\hline $\mathrm{Pt} / \mathrm{MoS}_{2} / \mathrm{Ti}$ & 1930 & $10 \sim 160$ & 0.87 & 7 \\
\hline $\mathrm{Pd} @ \mathrm{CeO}_{2}-\mathrm{SnO}_{2}$ & 472.4 & $146 \sim 1920$ & 0.044 & 8 \\
\hline np-PtCu & 64.7 & $10 \sim 1700$ & 0.1 & 9 \\
\hline Nf/Pd@Ag/rGO-NH & 1307.46 & $2 \sim 19500$ & 0.7 & 10 \\
\hline $\begin{array}{l}\text { Au-Pd bimetallic } \\
\text { supra-nanoparticles }\end{array}$ & $17.4(\mu \mathrm{A} / \mathrm{mM})$ & $1 \sim 10000$ & 1 & 11 \\
\hline
\end{tabular}


Table S2. Performance comparison of Pd Cubes, RDs and Octs towards glucose detection

\begin{tabular}{cccc}
\hline & $\begin{array}{c}\text { Sensitivity } \\
\left(\mu \mathrm{A} \mathrm{mM}^{-1} \mathrm{~cm}^{-2} \text { ECSA }\right)\end{array}$ & $\begin{array}{c}\text { Linear range } \\
(\mu \mathrm{M})\end{array}$ & $\begin{array}{c}\text { LOD } \\
(\mu \mathrm{M})\end{array}$ \\
\hline $\begin{array}{c}\text { Pd Cubes } \\
(100)\end{array}$ & 0.406 & $3-9999$ & 3 \\
Pd RDs & 0.183 & $15999-36999$ & 2 \\
$(110)$ & 0.558 & $2-9999$ & 3.6 \\
Pd Octs & 0.235 & $15999-42999$ & \\
$(111)$ & 0.324 & $4.2-9999$ & \\
\hline
\end{tabular}

Table S3. ECSA value of Pd cubes, RDs and Octs calculated by CO stripping curves

\begin{tabular}{cccc} 
& Pd cubes & Pd RDs & Pd Octs \\
\hline ECSA & & & \\
$\left(\mathrm{cm}^{2}\right)$ & 0.997 & 1.830 & 2.181 \\
\hline
\end{tabular}

\section{References}

1. Zhang, X.; Sheng, Q.; Zheng, J. Palladium nanoparticles decorated SnO2 wrapped MWCNT nanocomposites as a highly efficient H2O2 electrocatalyst. New Journal of Chemistry 2019, 43, 175-181.

2. Guler, M.; Turkoglu, V.; Kivrak, A.; Karahan, F. A novel nonenzymatic hydrogen peroxide amperometric sensor based on $\mathrm{Pd} @ \mathrm{CeO} 2-\mathrm{NH} 2$ nanocomposites modified glassy carbon electrode. Materials Science \& Engineering C-Materials for Biological Applications 2018, 90, 454-460.

3. Xue, W.; Bo, X.; Zhou, M.; Guo, L. Enzymeless electrochemical detection of hydrogen peroxide at Pd nanoparticles/porous graphene. Journal of Electroanalytical Chemistry 2016, 781, 204-211.

4. Mei, H.; Wu, W.; Yu, B.; Wu, H.; Wang, S.; Xia, Q. Nonenzymatic electrochemical sensor based on Fe@Pt core-shell nanoparticles for hydrogen peroxide, glucose and formaldehyde. Sensors and Actuators B-Chemical 2016, 223, 68-75.

5. Pang, P.; Yang, Z.; Xiao, S.; Xie, J.; Zhang, Y.; Gao, Y. Nonenzymatic amperometric determination of hydrogen peroxide by graphene and gold nanorods nanocomposite modified electrode. Journal of Electroanalytical Chemistry 2014, 727, 27-33.

6. Guan, H.; Zhang, J.; Liu, Y.; Zhao, Y.; Zhang, B. Rapid quantitative determination of hydrogen peroxide using an electrochemical sensor based on $\mathrm{PtNi}$ alloy/CeO2 plates embedded in $\mathrm{N}$-doped carbon 
nanofibers. Electrochimica Acta 2019, 295, 997-1005.

7. Zalneravicius, R.; Gedminas, A.; Ruzgas, T.; Jagminas, A. Nanoplatelet MoS2 arrays decorated with Pt nanoparticles for non-enzymatic detection of hydrogen peroxide. Journal of Electroanalytical Chemistry 2019, 839, 274-282.

8. Ensafi, A. A.; Mahmoodi, A.; Rezaei, B. Pd@CeO2-SnO2 nanocomposite, a highly selective and sensitive hydrogen peroxide electrochemical sensor. Sensors and Actuators B-Chemica/ 2019, 296. 9. Yang, H.; Wang, Z.; Zhou, Q.; Xu, C.; Hou, J. Nanoporous platinum-copper flowers for non-enzymatic sensitive detection of hydrogen peroxide and glucose at near-neutral $\mathrm{pH}$ values. Microchimica Acta 2019, 186.

10. Guler, M.; Turkoglu, V.; Bulut, A.; Zahmakiran, M. Electrochemical sensing of hydrogen peroxide using Pd@Ag bimetallic nanoparticles decorated functionalized reduced graphene oxide. Electrochimica Acta 2018, 263, 118-126.

11. Huang, Y.; Ferhan, A. R.; Dandapat, A.; Yoon, C. S.; Song, J. E.; Cho, E. C.; Kim, D.-H. A Strategy for the Formation of Gold-Palladium Supra-Nanoparticles from Gold Nanoparticles of Various Shapes and Their Application to High-Performance H2O2 Sensing. Journal of Physical Chemistry C 2015, $119,26164-26170$. 